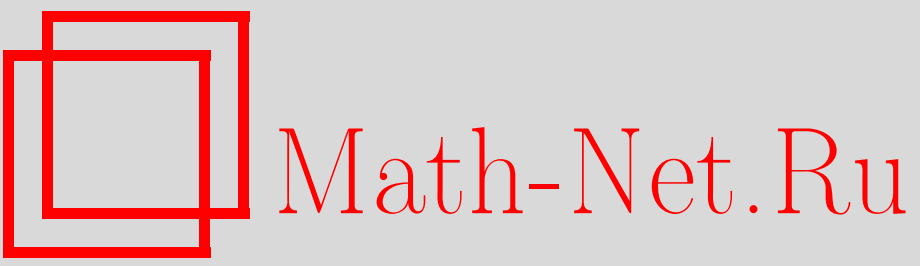

Н. П. Долбилин, М. А. Штанько, М. И. Штогрин, Неизгибаемость квадрильяжа тора, УМH, 1999, том 54, выпуск 4, 167-168

DOI: https://doi.org/10.4213/rm187

Использование Общероссийского математического портала Math-Net.Ru подразумевает, что вы прочитали и согласны с пользовательским соглашением

http://www.mathnet.ru/rus/agreement

Параметры загрузки:

IP: 54.198 .67 .100

26 апреля 2023 г., 17:12:01 


\title{
НЕИЗГИБАЕМОСТЬ КВАДРИЛЬЯЖА ТОРА
}

\author{
Н. П. ДольИлин, М. А. ШтАньКО, М. И. ШтоГРИн
}

Основные понятия и определения, использованные ниже, даны в работе [1], в которой была доказана неизгибаемость квадрильяжа сферы и сформулирована следующая гипотеза: квадрильяжное вложение сферы $\mathbb{Q}^{2}$ в $\mathbb{R}^{3}$ является решеточным.

Теорема. Пусть $\mathbb{Q}^{2}$ - произвольный квадрильяж сферы или тора. Тогда любое квадрильяжное вложение $f: \mathbb{Q}^{2} \rightarrow \mathbb{R}^{3}$, если оно существует, является решеточным.

При вложении $f: \mathbb{Q}^{2} \rightarrow \mathbb{R}^{3}$ для удобства прообраз $\mathbb{Q}^{2}$ отождествим с образом $f\left(\mathbb{Q}^{2}\right)$.

СледствиЕ. Квадрильяжное вложсение сферь или тора $\mathbb{Q}^{2}$ в $\mathbb{R}^{3}$ неизгибаемо.

Граф стыковки. Рассмотрим нерешеточное вложение $\mathbb{Q}^{2}$ в $\mathbb{R}^{3}$. Ребро из $\mathbb{Q}^{2}$ назовем $c m b$ ковочныц, если смежные по нему два квадрата из $\mathbb{Q}^{2} \subset \mathbb{R}^{3}$ образуют двугранный угол, не равный ни $\frac{\pi}{2}$, ни $\pi$. Все квадраты из $\mathbb{Q}^{2}$, сходящиеся в вершине $O$, составляют звезду St $O$. Выясним сколько радиусов звезды St $O$ могут быть стыковочными ребрами.

1. Вериина не может быть концом ровно одного стыковочного ребра. В самом деле, обходя всю звезду St $O$ вокруг $O$, начиная с радиуса i, получим один сектор $(O, \mathbf{i}, \mathbf{i})$. Если все внутренние радиусы сектора не являются стыковочными ребрами, то весь он вложен в одну кубическую решетку и, следовательно, один радиус $\mathbf{i}$ не может быть стыковочным ребром.

2. В вериине не могут сойтись ровно два максимально укрупненные стыковочные ребра. В самом деле, пусть в вершине сходится всего лишш два стыковочных ребра. Они разрезают звезду St $O$ на два сектора, каждьй из которых вложен в свою кубическую решетку. Вместе с каждым сектором оба ребра принадлежат обеим решеткам. Если они не коллинеарны, то они перпендикулярны друг другу, составляя пару вида $(\mathbf{i}, \mathbf{j})$. Тогда оба сектора $(O, \mathbf{i}, \mathbf{j})$ и $(O, \mathbf{j}, \mathbf{i})$ звезды $\mathrm{St} O$ вложены в одну кубическую решетку, и поэтому радиусы $\mathbf{i}$ и $\mathbf{j}$ не могут быть стыковочными ребрами. Остается единственная возможность: стыковочные ребра коллинеарны, имея вид $\mathbf{i},-\mathbf{i}$ (одно ребро лежит на продолжении другого), ввиду вложения $\mathbb{Q}^{2}$ в $\mathbb{R}^{3}$. Укрупним их до стыковочного диаметра звезды St $O$. Переходя от стыковочных ребер к максимально укрупненным стыковочным ребрам, мы избавимся от всех вершин, в которых сходятся точно два стыковочных ребра.

3. В вериине не могут сойтись ровно 3 стыковочные ребра. В самом деле, пусть в вершине $O$ сходится ровно 3 стыковочных ребра. Они разбивают звезду на 3 сектора, каждый из которых вложен в свою кубическую решетку. Каждая пара радиусов составляет угол $\frac{\pi}{2}$ или $\pi$. Среди трех углов, составленных тремя парами этих радиусов, не может быть двух углов равных $\pi$, иначе тройка радиусов не была бы вложена в $\mathbb{R}^{3}$. Значит, тройка углов имеет вид $\left(\frac{\pi}{2}, \frac{\pi}{2}, \frac{\pi}{2}\right)$ или $\left(\frac{\pi}{2}, \frac{\pi}{2}, \pi\right)$. В первом случае тройка стыковочных ребер составляет ортонормированньй репер $(\mathbf{i}, \mathbf{j}, \mathbf{k})$, все 3 сектора $(O, \mathbf{i}, \mathbf{j}),(O, \mathbf{j}, \mathbf{k}),(O, \mathbf{k}, \mathbf{i})$ вложены в одну кубическую решетку, ни одно из трех ребер $\mathbf{j}, \mathbf{k}, \mathbf{i}$ не является стыковочным. Во втором случае стыковочные ребра составляют тройку вида $(\mathbf{i}, \mathbf{j},-\mathbf{i})$, два сектора $(O, \mathbf{i}, \mathbf{j})$ и $(O, \mathbf{j},-\mathbf{i})$ вложены в одну кубическую решетку, разделяющее их ребро $\mathbf{j}$ не является стыковочным (радиусы -i и $\mathbf{i}$ сектора $(O,-\mathbf{i}, \mathbf{i})$ в общем случае задают стыковочный диаметр звезды $\mathrm{St} O)$. Следовательно, в вершине не может быть ровно 3 стыковочных ребра.

4. Если в вериине сходятся ровно 4 стыковочных ребра, то они составляют циклическую последовательность вида $(\mathbf{i}, \mathbf{j},-\mathbf{j},-\mathbf{i})$. В самом деле, 4 стыковочных ребра разделяют звезду St $O$ на 4 сектора. Угол между радиусами одного сектора равен либо $\frac{\pi}{2}$, либо $\pi$. Среди этих углов не может быть подряд двух углов равных $\pi$, в силу вложения $\mathbb{Q}^{2}$ в $\mathbb{R}^{3}$. Следовательно, для циклической последовательности из 4 углов между радиусами секторов имеются всего 3 возможности $\left(\frac{\pi}{2}, \frac{\pi}{2}, \frac{\pi}{2}, \frac{\pi}{2}\right),\left(\frac{\pi}{2}, \frac{\pi}{2}, \frac{\pi}{2}, \pi\right),\left(\frac{\pi}{2}, \pi, \frac{\pi}{2}, \pi\right)$.

В первом случае четверка радиусов имеет вид $(\mathbf{k}, \mathbf{i},-\mathbf{k}, \mathbf{i} \cos \alpha+\mathbf{j} \sin \alpha)$, где $0 \leqslant \alpha \leqslant \frac{\pi}{2}$. Два сектора $(O, \mathbf{k}, \mathbf{i})$ и $(O, \mathbf{i},-\mathbf{k})$ лежат в одной кубической решетке, их обшее ребро $\mathbf{i}$ не является

Работа выполнена при финансовой поддержке Российского фонда фундаментальных исследований (грант № 99-01-00010). 
ребром стыковки. Другие два сектора $(O,-\mathbf{k}, \mathbf{i} \cos \alpha+\mathbf{j} \sin \alpha)$ и $(O, \mathbf{i} \cos \alpha+\mathbf{j} \sin \alpha, \mathbf{k})$ также лежат в одной кубической решетке, их общее ребро $\mathbf{i} \cos \alpha+\mathbf{j} \sin \alpha$ не является ребром стыковки. Стьковочными ребрами могут быть лишь радиусы $\mathbf{k}$ и $-\mathbf{k}$ при $0<\alpha<\frac{\pi}{2}$, и после укрупнения $O$ будет внутренней точкой укрупненного стыковочного ребра.

Во втором случае 4 радиуса имеют вид $(\mathbf{i}, \mathbf{j}, \mathbf{k},-\mathbf{i})$. Три сектора $(O, \mathbf{i}, \mathbf{j}),(O, \mathbf{j}, \mathbf{k}),(O, \mathbf{k},-\mathbf{i})$ находятся в одной кубической решетке, и поэтому радиусы $\mathbf{j}, \mathbf{k}$ не являются стыковочными ребрами. Два радиуса - $\mathbf{i}$ и $\mathbf{i}$ сектора $(O,-\mathbf{i}, \mathbf{i})$ в общем случае дают стыковочный диаметр.

В третьем случае 4 радиуса имеют вид $(\mathbf{i}, \mathbf{j},-\mathbf{j},-\mathbf{i})$. Они разбивают звезду $\operatorname{St} O$ на 4 сектора $(O, \mathbf{i}, \mathbf{j}),(O, \mathbf{j},-\mathbf{j}),(O,-\mathbf{j},-\mathbf{i}),(O,-\mathbf{i}, \mathbf{i})$. Только в этом случае все 4 радиуса могут быть стыковочными ребрами.

ПримЕчАнИЕ. В последнем случае мы имеем вершину, в которой сходятся 4 максимально укрупненные стыковочные ребра, хотя каждое стыковочное ребро лежит на продолжении другого. Если в вершине сходятся не менее 4 стыковочных ребер, в окрестности этой вершины стыковочные ребра мы никогда не укрупняем, число стыковочных ребер в такой вершине всегда остается прежним.

5. Существует вершина, в которой сходится не менее 5 стыковочных ребер. В самом деле, в силу свойств $1,2,3$ и замкнутости поверхности $\mathbb{Q}^{2}$ максимально укрупненное стыковочное ребро имеет два конца, в каждом из которых сходится не меньше 4 стыковочных ребер. Если бы в каждой вершине сходилось ровно 4 стыковочных ребра, то прямая, на которой лежит одно стыковочное ребро, в силу свойства 4 продолжалась бы до бесконечности, что невозможно в силу замкнутости поверхности $\mathbb{Q}^{2}$. Итак, сушествует вершина, в которой сходится не менее 5 стыковочных ребер.

6. Граф, составленный из максимально укрупненных стыковочных ребер, назовем графом стыковки. После удаления из $\mathbb{Q}^{2}$ графа стыковки поверхность распадется на связные компоненты, край каждой из которых содержит не менее четырех ребер (так как край вместе с компонентой вложен в одну и ту же кубическую решетку, а в ней угол между выходящими из одного узла двумя ребрами равен $\frac{\pi}{2}$ или $\pi$ ), где под ребром понимается максимально укрупненное стыковочное ребро, т.е. ребро графа стыковки. В каждой вершине графа стыковки сходится не менее четырех его ребер. Связную компоненту впредь будем называть гранью.

ДоКАЗАТЕЛЬСТВО ТЕОРЕМЫ. Обозначим число вершин графа стыковки через 8 , число ребер через $p$, число граней через 2, эйлерову характеристику поверхности $\mathbb{Q}^{2}$ через $\chi$. Тогда по формуле Эйлера имеем $в-p+\imath \geqslant \chi$ (равенство достигается тогда, когда каждая грань гомеоморфна диску). Так как каждая грань имеет не менее 4 сторон, а в каждой вершине сходится не менее 4 граней, то $в=8_{4}+\theta_{5}+\theta_{6}+\cdots, 2 p=4 \theta_{4}+58_{5}+6 \theta_{6}+\cdots, 2=2_{4}+2_{5}+2_{6}+\cdots$, $2 p=42_{4}+52_{5}+62_{6}+\cdots$, где $8 q-$ число вершин, в которых сходятся по $q$ ребер, $2 n$ - число граней, ограниченных $n$ сторонами. Умножая эти равенства соответственно на $4,-1,4,-1$ и складывая их вместе, получим $4(8-p+2)=-8_{5}-2_{5}-28_{6}-2 \imath_{6}-3 \theta_{7}-3 \imath_{7}-\cdots$, или

$$
4 \chi \leqslant-\theta_{5}-2_{5}-2 \theta_{6}-2 \imath_{6}-3 \theta_{7}-32_{7}-\cdots .
$$

Это соотношение не может быть удовлетворено при $\chi=2$ и (с учетом свойства 5$)$ при $\chi=0$. Следовательно, в случае сферы или тора граф стьковки пуст. Теорема доказана.

ЗАмечАниЕ. Теорема справедлива не только в случае квадрильяжного вложения, но ив случае квадрильяжного отображения, при котором реберный остов квадрильяжа $\mathbb{Q}^{2}$, гомеоморфного сффере или тору, погружен в $\mathbb{R}^{3}$.

\section{СПИСОК ЛИТЕРАТУРЫ}

[1] Долбилин Н. П., Штанько М. А., Штогрин М. И. // Докл. АН. 1997. Т. 354. № 4. С. $443-445$. 